collected at each visit for virological analysis. Polymerase chain reaction assays for adenovirus, influenza $A \& B$, metapneumovirus, parainfluenza $1-3$, respiratory syncytial virus and rhinovirus were performed on each sample. Spirometry was recorded at each visit. Treatment failure was defined as a failure of the $\mathrm{FEV}_{1}$ to return to $\geq 90 \%$ of baseline after intravenous antibiotics. Statistical analysis utilised generalized linear models and multiple linear regression as appropriate, taking into account multiple observations from participants.

Results 191/626 (30.5\%) study visits were positive for $\geq 1$ virus with rhinovirus accounting for $72.5 \%$. The incidence of VRI and pulmonary exacerbation (PEx) was 1.6 and 2.5 cases/patient-year respectively. VRI was associated with increased risk of PEx (OR 2.2; 95\% CI $1.6-3.1 ; \mathrm{p}<0.001)$.

There was no significant difference in relative fall from baseline $\mathrm{FEV}_{1}$ at virus-positive compared with virus-negative visits (8.7 vs $9.4 \%, \mathrm{p}=0.4$ ). Acute fall in $\mathrm{FEV}_{1}$ was lower in virus-positive PEx compared with virus-negative PEx (12.7 vs $15.6 \%$; $p=0.04)$. Rate of PEx, but not of VRI, was associated with a statistically significant decline in $\mathrm{FEV}_{1}$ over one year, adjusted for age, sex and baseline lung function ( $\beta$ coefficient $-1.79 ; 95 \%$ CI -3.4 to $-0.2 ; p=0.02$ ).

Intravenous antibiotics were given for 122 PEx of which 90 had pre- and post-antibiotic $\mathrm{FEV}_{1}$ data available. 26/90 (29\%) were classified as treatment failures. There was a trend towards lower likelihood of treatment failure in virus-positive PEx (OR 0.55; 95\% CI 0.1 to $2.7 ; p=0.46$ ).

Conclusions Incidence of PEx, but not VRI, is associated with accelerated decline in FEV in adults with CF. Virus-positive PEx are associated with a lower acute fall in FEV than virus-negative PEx.

\section{P88 IS HYDROGEN CYANIDE A MARKER OF BURKHOLDERIA CEPACIA COMPLEX INFECTION?}

doi:10.1136/thoraxjnl-2012-202678.330

'FJ Gilchrist, 'H Simms, 'A Alcock, ${ }^{2} A M$ Jones, ${ }^{3} \mathrm{D}$ Smith, ${ }^{4} \mathrm{P}$ Spanel, ${ }^{2} \mathrm{AK}$ Webb, 'W Lenney. ' University Hospital of North Staffordshire, Stoke on Trent, UK; ${ }^{2}$ Manchester Adult Cystic Fibrosis Centre, Manchester, UK; ${ }^{3}$ Keele University, Keele, UK; ${ }^{4} \mathrm{~J}$ Heyrovsky Institute of Physical Chemistry, Prague, Czech Republic

Introduction Pseudomonas aeruginosa (PA) was thought to be the only organism found in the cystic fibrosis (CF) lung that produced hydrogen cyanide $(\mathrm{HCN})$. A recent study used a cyanide selective electrode to demonstrate HCN production by Burkholderia cepacia complex (BCC) when cultured under biofilm conditions. We replicated this in-vitro experiment using selected ion flow tube mass spectroscopy (SIFT-MS) as a more sensitive method of detecting $\mathrm{HCN}$ We also investigated $\mathrm{HCN}$ as an in-vivo marker of BCC infection.

Methods Twelve adults with CF were recruited as they had chronic BCC infection and were free from PA infection for $>12$ months. They provided mouth and nose exhalation breaths for $\mathrm{HCN}$ analysis and a sputum sample. The sputum sample was cultured and the isolated BCC recultured under planktonic (free floating) and biofilm (non-motile communities attached to glass beads) conditions. The HCN concentration in the headspace of the both culture types was measured after 24, 48, 72 and 96 hours of incubation. Sterile, control cultures were also analysed. Biofilm formation was assessed visually and with spectrophotometry after crystal violet staining. The mouth and nose exhaled breath from 10 patients with CF that were free from PA and BCC infection for $>12$ months was also analysed.

Results Biofilm formation was confirmed visually and using spectrophotometry: mean(SD) absorbance of crystal violet was $3.43(0.31)$ absorbance units (AU) in the biofilm cultures compared to $0.005(0.003) \mathrm{AU}$ in the planktonic and control cultures $(\mathrm{p}<0.001)$. At each of the 4 time points, the headspace HCN concentration was
$<10 \mathrm{ppbv}$ (equivalent to background levels) for all biofilm, planktonic and control cultures. The mean(SD) breath HCN concentrations were no higher in the subjects with chronic BCC infection than in subjects without BCC infection for mouth exhaled breath $(11.0(12.7) \vee 12.0(12.9)$ ppbv $p=0.87)$ and nose exhaled breath $(0.6(1.1) \vee 2.1(3.8) \mathrm{p}=0.23)$.

Conclusions Using SIFT-MS we did not identify elevated HCN concentrations in the headspace of BCC cultured under biofilm or planktonic conditions or in the breath of patients with chronic BCC infection. Therefore, $\mathrm{HCN}$ does not appear to be an in-vitro or in-vivo marker of BCC infection.

\section{P89 THE EFFECT OF SAMPLE HANDLING ON VIABLE BACTERIAL COMMUNITY PROFILES FROM CYSTIC FIBROSIS SPUTUM SAMPLES}

doi:10.1136/thoraxjnl-2012-202678.331

${ }^{1} \mathrm{TH}$ Hafiz, ${ }^{2} \mathrm{LC}$ Cuthbertson, ${ }^{2} \mathrm{AO}$ Oliver, ${ }^{3} \mathrm{~GB}$ Rogers, ${ }^{3} \mathrm{KD}$ Bruce, ${ }^{1} \mathrm{MP}$ Carroll, ${ }^{2} \mathrm{CV}$ van der Gast. 'Southampton University Hospital, Southampton, UK; ${ }^{2} N E R C$, Centre for EcologyHydrology, Wallingford, UK; ${ }^{3}$ Department of Life Sciences, King's College London, London, UK

Introduction Obtaining accurate information on the composition of Cystic fibrosis (CF) lung infections is essential for the selection of appropriate therapy and maintenance of respiratory function. Whilst significant advances have been made using molecular techniques to provide such characterisation, there is a pressing need to identify the most appropriate sampling handling protocols. Here, we investigated the relationship between the time period prior to sample freezing and the bacterial community profiles.

Methods Eleven sputum samples were collected from adult CF patients experiencing acute pulmonary exacerbations. Expectorated sputum was aliquoted into 12 equal portions, with one portion being frozen immediately and others transferred to $-80^{\circ} \mathrm{C}$ at time points over a 72 hour period. Samples were treated with propidium monoazide prior to DNA extraction to limit analysis to viable bacterial cells. The bacterial composition of samples was determined by $16 S$ rRNA gene Terminal Restriction Fragment Length Polymorphism (T-RFLP) profiling. Changes in bacterial community composition over the time course were then analysed using ecological statistical tools.

Results A marked change in the bacterial community composition and abundance was observed within the first hour. Using Bray Curtis similarity index, a $51 \%$ similarity change in the bacterial community was observed in the first hour when compared to the sample frozen immediately. Thereafter, the bacterial community remained relatively stable for the following 72 hours (Figure 1A). Species cumulative richness showed an increase of approximately 63 new bacterial species over 72 hours, of which 25/63 (40\%) emerged within the first hour. The mean sample richness also showed a significant increase in bacterial species within the first hour (Figure 1B).

Conclusion The data presented indicates a significant change in sample bacterial composition within the first hour of sample collection. These findings have clear implications for the handling of samples for viable community profiling.

\section{P90 OSTEOPOROSIS IN NON-CYSTIC FIBROSIS BRONCHIECTASIS (NCFBR) ADULTS}

doi:10.1136/thoraxjn-2012-202678.332

${ }^{1}$ SS Sunny, ${ }^{2} \mathrm{~A}$ Burns, ${ }^{1} \mathrm{H}$ Jary, ${ }^{3} \mathrm{~T}$ Aspray, ${ }^{1} \mathrm{~A}$ De Soyza. ${ }^{1}$ Sir William Leech Centre for Lung Research and Freeman Hospital Bronchiectasis Service, Freeman Hospital, Newcastle upon Tyne, UK; ${ }^{2}$ Bone Mineral Assessment, Freeman Hospital, Newcastle upon Tyne, UK; ${ }^{3} T$ The Bone Clinic, Freeman Hospital, Newcastle upon Tyne, UK

Introduction Whilst osteoporosis is associated with COPD, little is known on its prevalence in non-cystic fibrosis bronchiectasis 